\title{
Genome-scale modeling of carbon assimilation in Geobacillus icigianus
}

\author{
M. Kulyashov ${ }^{1 *}$, I. Akberdin ${ }^{1,2,3}$, A. Rozanov², S. Peltek ${ }^{2}$ \\ ${ }^{1}$ Novosibirsk National Research University, Novosibirsk, Russia \\ ${ }^{2}$ Institute of Cytology and Genetics SB RAS, Novosibirsk, Russia \\ ${ }^{3}$ Biology Department and Viral Information Institute, San Diego State University, San Diego, USA \\ *e-mail:m.kulyashov@mail.ru
}

Key words: genome-scale modeling, Geobacillus icigianus, metabolic pathways

Motivation and Aim: In the last decade, genome-scale modeling became a powerful and useful tool to investigate whole-cell microbial metabolism [1]. For model microbes, such as $E$. coli, a huge amount of experimental data on different hierarchical levels of cellular organization is a basis for the most detailed and complete metabolic reconstruction of the organism [2], while advances in high-throughput techniques and in theoretical approaches pave the way to explore metabolic potential of the organism for non-model microbial species. Here, we represent recently obtained theoretical and experimental results for Geobacillus icigianus, a thermophilic bacterium isolated from a hot spring three years ago.

Methods and Algorithms: G. icigianus was cultivated under the conditions of a bioreactor at $\mathrm{pH}=7$ with the constant rate of air supply, and in microbiological shakers at $\mathrm{pH}=8$. Measurements of culture growth were made using a spectrophotometer PE 5400, using a $1 \mathrm{ml}$ plastic cuvette to measure culture from a shaker and a $10 \mathrm{ml}$ glass cuvette for measuring culture from a bioreactor. To build the genome-scale model and its subsequent modifications, we harnessed a sequenced genome [NZ_JPYA00000000.1] and a set of tools [3-5].

Results: We conducted experiments on the cultivation of G. icigianus on various substrates and under different conditions. As a result, it was shown that the most effective for cultivation are the substrates: glucose and glycerine, and the optimal conditions: a temperature of $62{ }^{\circ} \mathrm{C}$ and $\mathrm{pH}=7$. To enable the growth of culture we needed to add salts of iron, magnesium, calcium, vitamins, nitrilotriacetic acid and trace elements into the media. The growth rate in experiments with bioreactor was equal to $0.5 \mathrm{~h}^{-1}$, while the theoretically predicted value of the growth rate was $0.66 \mathrm{~h}^{-1}$.

Conclusions: Initial results allowed us to determine the optimal growth parameters that will facilitate further experiments and also demonstrated that developed genome-scale model qualitatively corresponds to the data observed in the experiments. However, it still requires both refinement of the biomass equation and consideration of transcriptomic data for different growth conditions in order to quantitatively reproduce measured growth rate and make reliable predictions on the essentiality of diverse metabolic pathways.

\section{References}

1. Orth J.D., Thiele I., Palsson B.Ø. (2010) Nature biotechnology. 28(3):245.

2. Monk J.M. et al. (2017) Nature biotechnology. 35(10):904.

3. Overbeek R. et al. (2013) Nucleic acids research. 42(D1):D206-D214.

4. Arkin A.P. et al. (2016) bioRxiv, p.096354.

5. Kelley J.J. et al. (2017) Bioinformatics. 33(16):2596-2597. 